\title{
Avalanches of grains with inhomogeneous distribution of inner mass
}

\author{
M. Piva, ${ }^{1}$ R. G. Martino, ${ }^{2}$ J.-C. Géminard,${ }^{3}$ and M. A. Aguirre ${ }^{2, *}$ \\ ${ }^{1}$ Grupo de Medios Porosos, Facultad de Ingeniería, Universidad de Buenos Aires, Paseo Colón 850, 1063 Buenos Aires, Argentina \\ ${ }^{2}$ Grupo de Medios Porosos, Facultad de Ingeniería, Universidad de Buenos Aires, CONICET, Paseo Colón 850, \\ 1063 Buenos Aires, Argentina \\ ${ }^{3}$ Laboratoire de Physique, Ecole Normale Supérieure de Lyon, Université de Lyon, CNRS, 46 Allée d'Italie, 69364 Lyon Cedex 07, France
}

(Received 11 June 2018; published 12 December 2018)

\begin{abstract}
We study the stability of granular systems constituted by particles with inhomogeneous distribution of inner mass, which is a factor that has not been previously analyzed. In particular, we report experimental results concerning the stability of two-dimensional piles of hollow plexiglass cylinders containing an inserted smaller metal rod. These particles partially fill a rough-edged drum whose rotation produces successive avalanches. The distribution of the maximum angle of stability, the angle of repose, and the avalanche size are analyzed for systems in which the inserts are fixed or free to move. We reveal two interesting effects related to the nonuniform inner mass distribution. On the one hand, the disorder in the array of the center of mass, enhanced in the system of particles with a fixed insert, decreases the stability of the pile. On the other hand, both mobile or fixed inserts lead to larger erosion of the free surface once the avalanche is triggered.
\end{abstract}

DOI: 10.1103/PhysRevE.98.062902

\section{INTRODUCTION}

The mechanical stability of granular piles has been the subject of numerous studies, both experimental and theoretical [1-12], and it has been found to depend on various factors: the number of grains, packing fraction, history, boundary conditions, and cohesion, particularly due to humidity. Also, the maximum slope that the pile can exhibit depends on the shape of the grains, on the physical properties of their surface (for instance on the roughness), and on the distribution of their size [13-17].

Many experiments have been carried out with symmetrical grains, e.g., spheres. Grains with a radial symmetry have a tendency to organize in crystals characterized by an ordered contact network as well as an ordered positional array of the center of mass of the particles. It has been observed that such ordered and compact arrays of grains are more stable than disordered ones [6]. The formation of crystals can be avoided by the use of nonspherical grains. In the absence of crystallization, the stability of the granular pile is not only influenced by the disorder, but also by the packing fraction, by the geometry of the grains, and by the roughness of the grain surface $[13,14,16]$.

For instance, Cantelaube et al. [13] have studied the stability of piles of pentagons and polydisperse disks. In both cases, piles are amorphous or disordered in the sense that they lack crystalline order. Nevertheless, the pile of pentagons has been observed to be much more stable than the pile of disks. The observed behavior is explained by the face-to-face contacts between the pentagons, which restrict the rotations and, thus, enhance the stability.

\footnotetext{
*Corresponding author: maaguir@fi.uba.ar
}

Another example was provided by Ertaş et al. [14] and Olson et al. [16], who studied the stability of two-dimensional piles of anisotropic or elongated grains. In particular, Ertaş et al. have studied piles of monodisperse glass spheres (monomers) mixed with a fraction of dimers (two glass spheres rigidly bonded), i.e., short elongated grains with the same typical surface irregularities as the spherical grains. The increase in the weight fraction of dimers leads to a decrease in the packing fraction, but to an increase in the stability of the pile: the stability of the pile is related to the irregularity of the dimer shape at the grain scale, which enhances their stability at the free surface. In addition, Olson et al. have also considered, experimentally and numerically, monomers, dimers, and trimers, and they reported the effect of grain shape on the ordering and stability of the piles. They found that dimers form piles that are much more stable than both of the other systems. Piles of trimers are the most unstable because they are the most disordered (in both position and contact networks), with the grains failing to form an ordered network. However, monomers are unstable with respect to shearing motion along the lattice directions, while dimers are more stable because shearing motion is inhibited by the disordered arrangement of the dimer bonds that enhances interlocking.

Finally, we mention that Frette et al. analyzed the avalanche dynamics for piles of three types of grains of rice: elongated rough grains, elongated smooth grains, and rounded smooth grains [18]. They observed that elongated grains slide collectively while rounded grains tend to roll individually. Even if the authors have not specifically analyzed the stability of the piles, one can guess that these different relaxation mechanisms might influence the stability. They observed a smaller angle of repose, which indicates a less stable system, for the pile of rounded grains.

In many practical situations, particles might have an inhomogeneous distribution of their inner mass. This is the case, 


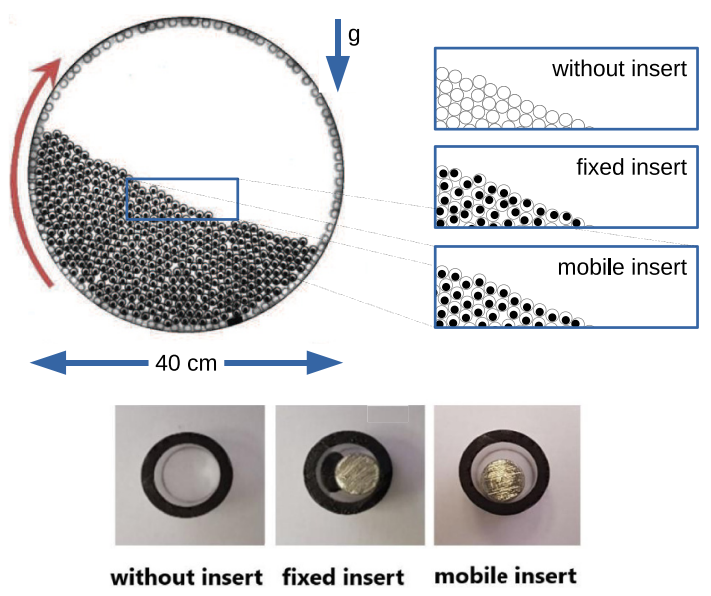

FIG. 1. Top left: picture of the experimental setup. The cylindrical drum (inner diameter $40 \mathrm{~cm}$ ) is filled with cylinders with a mobile insert, i.e., a steel rod. Top right: sketch of the three different types of piles. Bottom: photographs of the three types of grains (external diameter $9.8 \mathrm{~mm}$ ).

for instance, with seeds and with pharmaceutical capsules partially filled with powder. In this case, the center of mass of the particles does not correspond to their geometrical center. Moreover, for instance in the case of pharmaceutical capsules, the possible motion of the inner powder introduces an internal degree of freedom, i.e., a mobile center of mass. Therefore, it is pertinent to analyze the stability of piles of grains having a center of mass displaced from their geometrical center. In the present work, we study the stability of two-dimensional granular systems constituted by hollow plexiglass cylinders containing a smaller steel rod.

In particular, the following three systems are considered (Fig. 1):

(i) A pile of hollow plexiglass cylinders containing a smaller rod that is free to roll inside the cylinder: at rest, inserts are at their lowest position inside the cylinders, leading to systems that can present order in the contact network as well as in the array of centers of mass. However, during avalanches, the center of mass is free to move inside the particle.

(ii) A pile of hollow plexiglass cylinders containing a smaller solid metal rod that is fixed to the inner wall of the cylinder: at rest, this system can exhibit order of the contact network without any order of the centers of mass.

(iii) A pile of axisymmetric particles, cylinders without an insert, which is taken as a reference case.

We remark, in addition, that even for piles of axisymmetric particles, i.e., two-dimensional stacks of homogeneous and isotropic cylinders, not much work has previously been done regarding their stability $[5,6,13]$.

\section{EXPERIMENTAL SETUP}

The experimental setup is sketched in Fig. 1. A total of 300 cylindrical particles $(N=300)$ partially fill a cylindrical drum (inner diameter $D_{\text {drum }}=40.0 \pm 0.1 \mathrm{~cm}$ and width $L_{\text {gap }}=15.0 \pm 0.1 \mathrm{~mm}$ ) with a horizontal axis of rotation. To prevent the grains from organizing into a hexagonal array, a

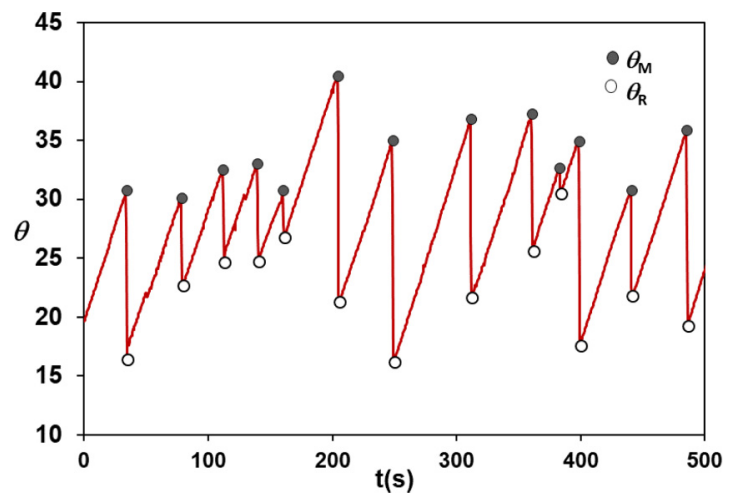

FIG. 2. Angle $\theta(t)$ vs time $t$ for a system of particles without an insert.

partial monolayer of particles is glued to the inner wall of the drum in a disordered manner. The two vertical walls are transparent, which makes it possible to image the grains from the side.

Each grain consists of a cylindrical external shell of length $L=15.00 \pm 0.02 \mathrm{~mm}$, external diameter $D=9.80 \pm$ $0.02 \mathrm{~mm}$, inner diameter $d=7.00 \pm 0.02 \mathrm{~mm}$, and mass $m_{\text {cyl }}=0.63 \pm 0.03 \mathrm{~g}$. Depending on the experimental conditions, the shell is empty (grains without an insert) or a steel rod (the insert) of length $l=14.2 \pm 0.1 \mathrm{~mm}$, diameter $d_{s}=5.00 \pm 0.02 \mathrm{~mm}$, and mass $m_{\mathrm{rod}}=2.14 \pm 0.02 \mathrm{~g}$ is introduced inside. The insert is either free to move (mobile insert) or fixed (fixed insert). For the grains with a mobile insert, the center of mass is displaced at most, when the insert is in contact with the inner wall of the shell, by $d_{\text {c.m. }}=$ $0.77 \pm 0.05 \mathrm{~mm}$, i.e., $d_{\text {c.m. }} \cong 0.16 \mathrm{D} / 2$, from the axis of the plexiglass shell. For the grains with a fixed insert, the metal rod is maintained against the inner wall of the shell by two rubber beads of diameter $d_{\text {bead }}=3.00 \pm 0.02 \mathrm{~mm}$ and mass $m_{\text {bead }}=0.030 \pm 0.005 \mathrm{~g}$, such that, due to their additional mass, the center of mass of the assembly is displaced by $d_{\text {c.m. }}=0.76 \pm 0.05 \mathrm{~mm}$ from the axis. Photographs of the grains are presented in Fig. 1.

Successive independent avalanches are produced, approximately every $\tau_{\mathrm{av}}=13 \pm 5 \mathrm{~s}$, by rotating the drum at small angular velocity, $\omega=0.85 \mathrm{deg} / \mathrm{s}$ [19]. An avalanche is a catastrophic event that affects the free surface and a few layers beneath it, leading to a sudden decrease of the slope of the pile over a time of typically $\tau=1 \mathrm{~s}$, much smaller than $\tau_{\text {av }}$. The frame rate does not make it possible to resolve the dynamics during the avalanche, but it is sufficient to assess the time between two successive avalanches $\tau_{\mathrm{av}}$ to within $0.5 \mathrm{~s}$. Avalanches can be characterized by the maximum angle of stability, $\theta_{M}$, which is the slope that the free surface makes with the horizontal when the avalanche starts, and by the angle of repose, $\theta_{R}$, which is the slope that the free surface makes with the horizontal when the avalanche stops.

To determine both characteristic angles, $\theta_{M}$ and $\theta_{R}$, we report the angle $\theta(t)$ that the free surface makes with the horizontal as a function of time $t$ (Fig. 2): the pile side is imaged with a monochrome CCD camera, at $2 \mathrm{~Hz}$. For each image, the free surface coordinates are found with ImageJ [20] and the angle $\theta$ is obtained by linear interpolation with a precision of 0.2 deg. In Fig. 2, an avalanche is characterized by a sudden 
TABLE I. Average values of $\theta_{M}, \theta_{R}$, and $\delta$.

\begin{tabular}{lccc}
\hline \hline Type of packing & $\left\langle\theta_{M}\right\rangle(\mathrm{deg})$ & $\left\langle\theta_{R}\right\rangle(\mathrm{deg})$ & $\langle\delta\rangle(\mathrm{deg})$ \\
\hline Fixed insert & $32 \pm 4$ & $20 \pm 4$ & $12 \pm 6$ \\
Without insert & $33 \pm 4$ & $23 \pm 4$ & $10 \pm 5$ \\
Mobile insert & $34 \pm 4$ & $23 \pm 4$ & $11 \pm 6$ \\
\hline \hline
\end{tabular}

decrease of $\theta$. Indeed, before an avalanche, $\theta(t)$ increases linearly until the system loses stability at the maximum angle $\theta_{M}$. Because the angular velocity $\omega$ is small, the sudden decrease of the slope associated with the rearrangements of the grains is easily detected.

Finally, we mention that, even if the spatial resolution is good enough to determine precisely the angle $\theta(t)$, the imaging system does not allow the tracking of the insert inside the shell. In the following, we thus limit our study to the global effects of the insert on the angle of avalanche, $\theta_{M}$, and the angle of repose, $\theta_{R}$, without considering the internal state of the system.

\section{EXPERIMENTAL RESULTS}

We report the results of experimental runs during which more than 700 avalanches were detected. We consider that an avalanche corresponds to a sudden decrease of $\theta(t)$ by more than $1 \mathrm{deg}$. Note that, with such a threshold, small rearrangements that occur before an avalanche and do not significantly alter the slope (drop in $\theta$ smaller than $1 \mathrm{deg}$ ) are naturally filtered by this procedure and thus discarded from this study.

For each avalanche, we measure, as illustrated in Fig. 2, the angle of avalanche, $\theta_{M}$, and the angle of repose, $\theta_{R}$. Both angles fluctuate throughout the experimental run, and it is thus adequate to discuss their values through their probability density functions (PDFs). We point out that the PDFs reveal a large dispersion. To exclude that the dispersion was not due to a lack of statistics, we performed a complementary longer experiment that has not revealed any substantial difference in the width of the distribution or mean values of $\theta_{M}$ and $\theta_{R}$. The dispersion is thus intrinsic to this type of system, as was also observed in the two-dimensional experimental work of Cantelaube et al. [13]. Taking into account that the uncertainty in the value of both angles is about $1 \mathrm{deg}$, we shall report histograms using bins of $2 \mathrm{deg}$.

\section{A. Characteristic angles}

We focus first on the stability of the pile, thus on the angle of avalanche, $\theta_{M}$. PDFs and average values of $\theta_{M}$ for the three systems under study (grains without or with an insert, fixed or mobile) are presented in Fig. 3 and Table I, respectively. For all systems, the distributions are skewed to larger values of $\theta$, but, regarding the stability, the following observations can be made:

(i) The pile of particles with a fixed insert is associated with the smallest average value $\left\langle\theta_{M}\right\rangle$ of $\theta_{M}$. This system is thus the most unstable, on average.

(ii) The pile of particles with a mobile insert is associated with the largest average value $\left\langle\theta_{M}\right\rangle$ of $\theta_{M}$. This system is thus

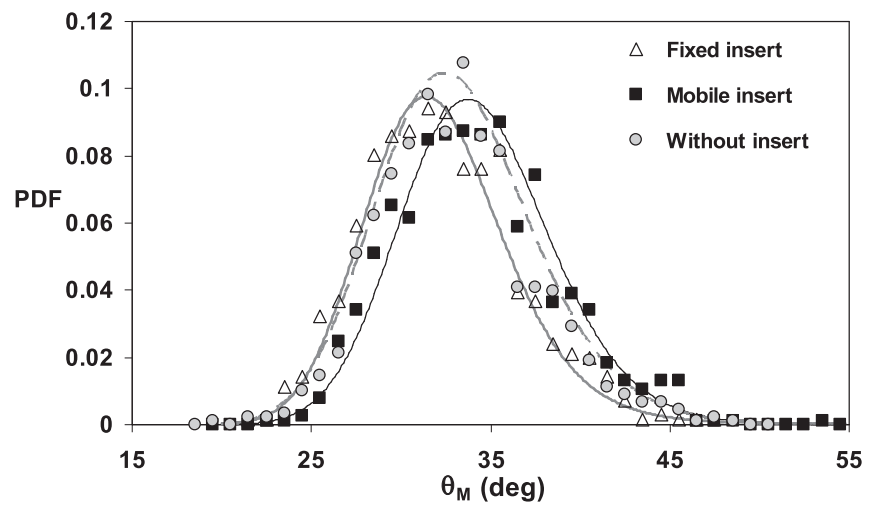

FIG. 3. PDF of the angle of avalanche $\theta_{M}$. Markers are placed at the center of each bin ( $2 \mathrm{deg}$ ). Lines are only a guide for the eye.

the most stable, even more stable on average than the pile of grains without an insert.

Let us now consider the state of the system right after the avalanche, which is, at least partially, characterized by the angle of repose, $\theta_{R}$. We observe on the PDFs reported in Fig. 4 that, in all cases, the distributions are skewed to larger values of $\theta$, as previously observed for $\theta_{M}$. From the PDFs in Fig. 4 and from the average values reported in Table I, we note the following:

(i) Avalanches of particles without an insert or with a mobile insert stop, on average, when the slope of the free surface reaches almost the same angle of repose, $\theta_{R}$.

(ii) Avalanches of particles with a fixed insert lead to a significantly smaller angle of repose, $\theta_{R}$.

\section{B. Avalanche size}

Before we discuss the qualitative effects of the insert on $\theta_{M}$ and $\theta_{R}$, we report on measurements of the avalanche size in term of the variation $\delta \equiv \theta_{M}-\theta_{R}$ of the free surface slope resulting from the avalanche. From the PDFs of $\delta$ reported in Fig. 5 and from the average values reported in Table I, we draw the following conclusions:

(i) Avalanches of particles with a fixed insert are associated with the largest values of $\delta$, thus with the largest variation of the slope of the free surface.

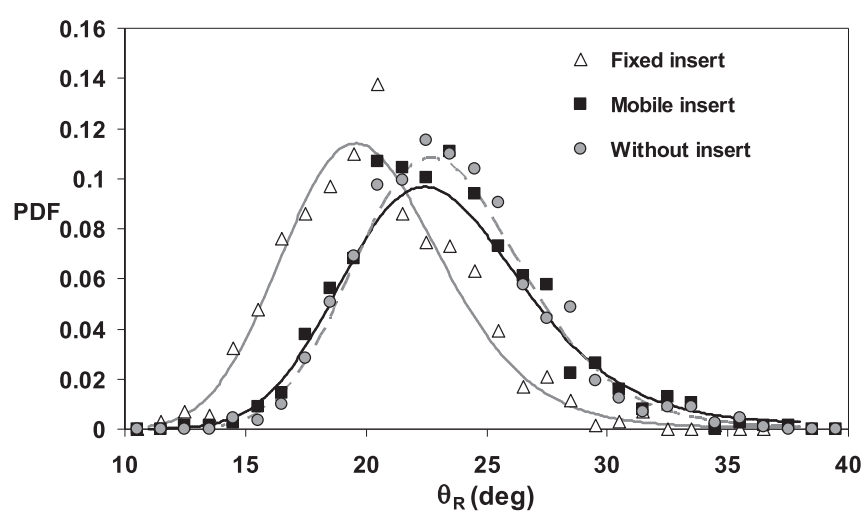

FIG. 4. PDF of the angle of repose $\theta_{R}$. Markers are placed at the center of each bin $(2 \mathrm{deg})$. Lines are only a guide for the eye. 


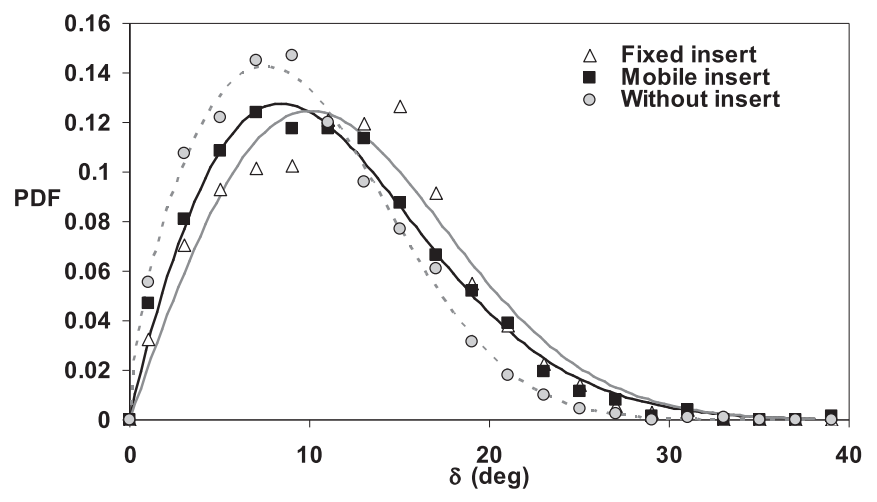

FIG. 5. PDF of the avalanche amplitude $\delta$. Markers are placed at the center of each bin $(2 \mathrm{deg})$. Lines are only a guide for the eye.

(ii) Avalanches of particles without an insert are associated with the smallest values of $\delta$.

One can then wonder if the size of the avalanche in terms of the variation of the slope of the free surface, $\delta$, corresponds to its size in terms of the displacement of the grains during an event. To get some insights, we measure the cumulative displacement of grains in relation to the cumulative size of the 300 particles, Fm. To do so, each frame is binarized (with the same threshold) to detect the proportion of black pixels, $P o$, that corresponds to the 300 particles in the drum. From the fluctuations observed from one image to another, we estimate that $\mathrm{Po}$ is measured to within $1 \%$. Then, excluding the rotation of the drum, the difference between two consecutive frames gives an image in which the fraction of black pixels, $P m$, accounts for the cumulative displacement of the particles. For each avalanche $P_{\max }$, the maximum value of $P m$ is measured, and we define $F m \equiv P_{\max } / P o$. Considering the uncertainty in the determination of $\mathrm{Pm}$ (and $\mathrm{Po}$ ), we estimate that $F m$ is obtained with at most $3 \%$ uncertainty.

One could expect that the amplitude $\delta$ (the change in the slope) is proportional to the avalanche size in terms of the cumulative displacement of the grains that move. We indeed observe a good correlation between $\delta$ and $F m$, which are, on average, proportional to one another for each type of grains (Fig. 6). However, the proportional coefficient is systemdependent: for the same change in the slope $\delta$, avalanches of the particles without an insert involve more grains than avalanches of both of the other systems, whereas avalanches

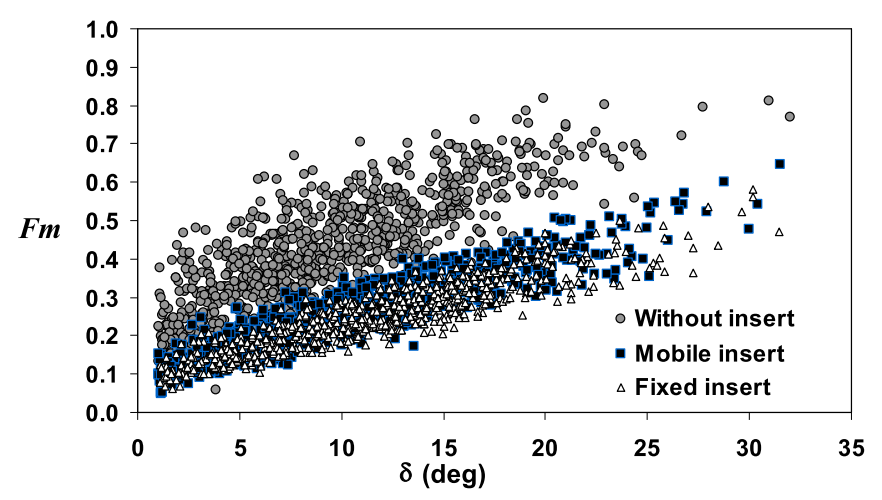

FIG. 6. Avalanche relative size $F m$ vs amplitude $\delta$.

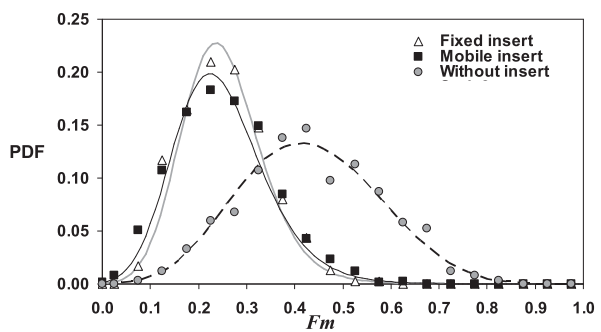

FIG. 7. PDF of the avalanche size Fm. Markers are placed at the center of each bin (0.05). Lines are only a guide for the eye.

of grains with mobile or fixed inserts are of the same size. In conclusion, the avalanches of grains without an insert involve on average a significantly larger cumulative displacement of grains, as observed in the PDFs of Fm reported in Fig. 7.

\section{DISCUSSION AND CONCLUSIONS}

We studied the stability of 2D piles of grains considering the angle of avalanche $\theta_{M}$, the angle of repose $\theta_{R}$, and the size of the avalanche in terms of both the variation $\delta$ of the free surface slope and the cumulative displacement of the grains involved, Fm. Grains without or with inserts, fixed or mobile, were considered.

First, we point out that the effects of the insert on the characteristic angles, $\theta_{M}$ and $\theta_{R}$, remain small: the angle of avalanche, $\theta_{M}$, and the angle of repose, $\theta_{R}$, are changed by two or three degrees, at most (Table I). Accordingly, the effect of the insert on the avalanche size, characterized in terms of the variation $\delta$ of the inclination of the free surface, remains small (Fig. 5). However, we observe that the insert has a rather large effect on the displacement of the grains involved in the avalanche (Fig. 7): avalanches of grains without an insert involve a significantly larger displacement. Remarking that the grains with an insert, fixed or mobile, are significantly heavier than the grains without an insert, we conclude that the avalanche size in terms of cumulative displacement of grains is altered by the weight of the particles only and not by the additional internal degree of freedom added by the insert.

We observe that the particles with a fixed insert form the less stable piles (smallest $\theta_{M}$, Fig. 3) and give rise to the avalanches with the largest erosion (largest $\delta$, Fig. 5). The angle of avalanche relies on the fact that, upon tilting, a local configuration of particles loses its mechanical stability. Considering the latter, the effect of the fixed insert on the stability of the pile can be understood by the fact that the stability of the local arrangement of the particles depends on the angular orientation of the insert. In particular, if oriented toward the slope, the insert adds a moment of the weight about the contact point that favors the loss of stability. Due to the disorder in the angular position of the insert in the pile, upon tilting, the system reaches such an unstable configuration and loses stability for an angle $\theta_{M}$, smaller, on average, than observed for the two other systems. Thus, some configurations of grains that would be stable for grains without an insert or with a mobile insert are unstable for grains with a fixed insert. We can thus presume, expressing this feature in a different way, that the fixed insert reduces the number of stable 
configurations accessible to the system. Therefore, from such a viewpoint, we can infer why particles with a fixed insert give rise to the largest erosion (largest $\delta$ ): for the avalanche to end, the system must encounter a new stable configuration, and due to the reduction in the number of those configurations the avalanche lasts longer before a new stable state is reached, i.e., a configuration of the geometrical center of the particle and of the angular position of the insert that satisfies the mechanical stability.

By contrast, particles with a mobile insert form the most stable piles (Fig. 3) with intermediate erosion (Fig. 5). Upon tilting, the insert rolls at its lowest position inside the particle. In this position, the center of mass of the particles remains closer to the contact points below, and, as a consequence, the moment of the weight, which is reduced, is less likely to overcome the friction. This system is even more stable than the system without an insert. During the avalanche, the insert moves inside the grains, having its own kinetic energy: a particle (its shell) can encounter a stable position, but the inner motion of the insert can destabilize this position of the particle, which then goes farther [21]. As a consequence, the variation of the slope, $\delta$, is slightly larger for grains with a mobile insert than for the particles without an insert.
The present study reveals the interesting effects of the inhomogeneous distribution of the inner mass in particles constituting a granular material. This work opens a much wider field of investigation. On the one hand, one can consider the equilibrium and flow properties of granular piles made of particles having a continuous, inhomogeneous distribution of mass or a mobile insert with dissipation (viscous or solid friction, using, for instance, a powder as an insert) or several discrete permitted positions (sawtooth or ratchet on the inner wall of the external shell). On the other hand, one can regard an array of grains with a fixed insert as a prototype of plastic crystal, a material likely to exhibit a crystalline order of the geometrical center of the particles but an orientational disorder at the same time. The study of this system is particularly interesting in the framework of the statistical mechanics of dissipative systems.

\section{ACKNOWLEDGMENTS}

This work has been supported by the ANPCyT (Argentina) through Grant No. PICT-2014-2587, and the Centro Argentino Francés de Ciencias de la Ingeniería (CAFCI, CONICET-CNRS) through Grant No. R147-15.
[1] V. U. Nguyen and R. N. Chowdhury, Probabilistic study of spoil pile stability in strip coal mines-Two techniques compared, Int. J. Rock Mech. Mining Sci. Geomech. Abs. 21, 303 (1984).

[2] M. Aguirre, A. Calvo, and I. Ippolito, Avalanche Dynamics: Influence of the Granular Packing Size, MRS Proc. No. 627 (Materials Research Society, Pittsburgh, PA, 2000), p. BB2.3.

[3] M. A. Aguirre, N. Nerone, and A. Calvo, Influence of the number of layers on the equilibrium of a granular packing, Phys. Rev. E 62, 738 (2000).

[4] N. Nerone, M. A. Aguirre, A. Calvo, D. Bideau, and I. Ippolito, Instabilities in slowly driven granular packing, Phys. Rev. E 67, 011302 (2003).

[5] M. A. Aguirre, A. Medus, M. Mancuso, A. Calvo, and I. Ippolito, Effect of spontaneous rearrangements in the disorder of packing of discs, Physica A 371, 29 (2006).

[6] M. A. Aguirre, A. Calvo, I. Ippolito, A. Medus, and M. Mancuso, Rearrangements in a bidimensional packing of discs, Phys. Rev. E 73, 041307 (2006).

[7] X. Y. Liu, E. Specht, and J. Mellmann, Experimental study of the lower and upper angles of repose of granular material, Powder Technol. 154, 125 (2005).

[8] P. Evesque, D. Fargeix, P. Habib, M. P. Luong, and P. Porion, Pile density is a control parameter of sand avalanches, Phys. Rev. E 47, 2326 (1993).

[9] R. Fischer, P. Gondret, B. Perrin, and M. Rabaud, Dynamics of dry granular avalanches, Phys. Rev. E 78, 021302 (2008).

[10] R. Albert, I. Albert, D. Hornbaker, P. Schiffer, and A.-L. Barabási, Maximum angle of stability in wet and dry spherical granular media, Phys. Rev. E 56, R6271 (1997).
[11] N. Frayssea, H. Thomé, and L. Petit, Humidity effects on the stability of a sandpile, Eur. Phys. J. B 11, 615 (1999).

[12] S. Nowak, A. Samadani, and A. Kudrolli, Maximum angle of stability of a wet granular pile, Nat. Phys. 1, 50 (2005).

[13] F. Cantelaube, Y. Limon-Duparcmeur, D. Bideau, and G. H. Ristow, Geometrical analysis of avalanches in a $2 \mathrm{D}$ drum, J. Phys. (France) I 5, 581 (1995).

[14] D. Ertaş, T. C. Halsey, A. J. Levine, and T. G. Mason, Stability of monomer-dimer piles, Phys. Rev. E 66, 051307 (2002).

[15] S. Courrech du Pont, P. Gondret, B. Perrin, and M. Rabaud, Wall effects on granular heap stability, Europhys. Lett. 61, 492 (2003).

[16] C. J. Olson, C. Reichhardt, M. McCloskey, and R. J. Zieve, Effect of grain anisotropy on ordering, stability and dynamics in granular systems, Europhys. Lett. 57, 904 (2002).

[17] J. Olson, M. Priester, J. Luo, S. Chopra, and R. J. Zieve, Packing fractions and maximum angles of stability of granular materials, Phys. Rev. E 72, 031302 (2005).

[18] V. Frette, K. Christensen, A. Malthen-Sørenssen, J. Feder, T. Jøssang, and P. Meakin, Avalanche dynamics in a pile of rice, Nature (London) 379, 49 (1996).

[19] J. Rajchenbach, Flow in Powders: From Discrete Avalanches to Continuous Regime, Phys. Rev. Lett. 65, 2221 (1990).

[20] C. A. Schneider, W. S. Rasband, and K. W. Eliceiri, NIH image to ImageJ: 25 years of image analysis, Nat. Methods 9, 671 (2012).

[21] See Supplemental Material at http://link.aps.org/supplemental/ 10.1103/PhysRevE.98.062902 for a video of an avalanche of particles with a mobile insert. The video is sampled at $300 \mathrm{~Hz}$, a larger sampling frame rate than the one used in the experiments (2 Hz). 